\title{
Desenvolvimento de um software para suporte à avaliação fisioterápica baseado na Classificação Internacional de Funcionalidade, Incapacidade e Saúde
}

\author{
Development of a software for supporting the physiotherapeutic evaluation \\ based on the International Classification of Functioning, Disability and Health \\ Desarrollo de un software para apoyar a la evaluación fisioterápica basado en la \\ Clasificación Internacional del Funcionamiento, de la Discapacidad y de la Salud
}

Basílio Henrique Pereira Júnior | basiliofisioterapeuta@gmail.com

Centro Universitário de João Pessoa. João Pessoa, Brasil.

Maria Elma de Souza Soares Maciel | elma.smaciel@gmail.com

Centro Universitário de João Pessoa. João Pessoa, Brasil.

Walace Sartori Bonfim | walace.bonfim@fabrica.unipe.br

Centro Universitário de João Pessoa. João Pessoa, Brasil.

Mariana de Brito Barbosa | mariana@unipe.br

Centro Universitário de João Pessoa. João Pessoa, Brasil.

Juliana da Costa Santos Pessoa |jullycs.fisio@gmail.com

Centro Universitário de João Pessoa. João Pessoa, Brasil.

\section{Resumo}

Este relato, de caráter exploratório, é resultado de um projeto interdisciplinar entre acadêmicos dos cursos de fisioterapia e ciência da computação e tem como objetivo promover o uso da Classificação Internacional de Funcionalidade, Incapacidade e Saúde (CIF), por meio de um software avaliativo denominado CIFisio. Este software atende à área de fisioterapia ortopédica e traumatológica funcionando como gerenciador dos dados dos usuários do serviço de saúde. O sistema foi criado para ser utilizado pelos acadêmicos para avaliação e tratamento fisioterapêutico, registro de condutas diárias, reavaliações e geração de relatórios estatísticos descritivos, de modo a auxiliar a elaboração dos modelos de gestão e decisão. Acredita-se que o resultado da pesquisa aqui apresentada contribuirá com o processo de informatização em saúde, com a qualidade da assistência prestada pelos fisioterapeutas e aperfeiçoamento das práticas pedagógicas utilizadas na formação profissional de acadêmicos na área de saúde.

Palavras-chave: informática em saúde; informática clínica; sistemas de informação; Classificação Internacional de Funcionalidade, Incapacidade e Saúde; avaliação da deficiência. 


\begin{abstract}
This exploratory research is an interdisciplinary project between academics and students of the courses of physiotherapy and computational science and has as its objective promoting the use of the International Classification of Functioning, Disability and Health (ICF) by means of an evaluative software called CIFisio. This software supports the area of orthopedic and traumatological physiotherapy working as a data manager of the users of health services. The system was created to be used by the academics and students for physiotherapeutic evaluation and treatment, registry of daily conduct, new evaluations and to generate statistic descriptive reports, so that it aids the elaboration of models of management and decision. We believe that the result of this research will contribute to the process of informatization in health, to the quality of the assistance provided by the physiotherapists and the improvement of the pedagogical practices in the professional formation of students in the area.
\end{abstract}

Keywords: healthcare informatics; clinical informatics; information systems; International Classification of Functioning, Disability and Health; deficiency evaluation.

\title{
Resumen
}

Esta investigación, de carácter exploratorio, es un proyecto interdisciplinario entre académicos de los cursos de fisioterapia y ciencia de la computación y tiene como objetivo promover el uso de la Clasificación Internacional del Funcionamiento, de la Discapacidad y de la Salud (CIF), a través de un software de evaluación denominado CIFisio. Este software atiende al área de fisioterapia ortopédica y traumatológica funcionando como gestor de los datos de los usuarios del servicio de salud. El sistema fue creado para ser utilizado por los académicos para evaluación y tratamiento fisioterapéutico, registro de conductas diarias, nuevas evaluaciones y generación de informes estadísticos descriptivos auxiliando en los modelos de gestión y decisión. Se cree que el resultado de esta investigación contribuirá con el proceso de informatización en salud, calidad de la asistencia prestada por los fisioterapeutas y perfeccionamiento de las prácticas pedagógicas en la formación profesional de académicos en el área de salud.

Palabras clave: informática en salud; informática clínica; sistemas de información; Clasificación Internacional de Funcionamiento, Discapacidad y Salud; evaluación de la deficiencia.

Contribuição dos autores: os autores são responsáveis pelo texto.

Declaração de conflito de interesses: não há.

Fontes de financiamento: Fábrica de Softwares do Centro Universitário de João Pessoa - UNIPÊ.

Considerações éticas: não há.

Agradecimento/Contribuições adicionais: não há.

Histórico do artigo: Submetido: 04.out.2016 | Aceito: 05.jun.2017 | Publicado: 29.dez.2017

Apresentação anterior: não houve.

Licença CC BY-NC atribuição não comercial. Com essa licença é permitido acessar, baixar (download), copiar, imprimir, compartilhar, reutilizar e distribuir os artigos, desde que para uso não comercial e com a citação da fonte, conferindo os devidos créditos de autoria e menção à Reciis. Nesses casos, nenhuma permissão é necessária por parte dos autores ou dos editores. 


\section{Introdução}

No Brasil, a fundamentação de uma Política Nacional de Informação e Informática em Saúde (PNIIS) foi deliberada em dezembro de 2003, durante a $12^{\mathrm{a}}$ Conferência Nacional de Saúde. Este processo está em sintonia com o contexto internacional, que vem destacando a relevância de políticas e estratégias setoriais de comunicação e informação em saúde, gerando novos processos, produtos e mudanças nos modelos institucionais de gestão. Essa política é relevante nesse processo de informatização, tanto na assistência prestada individualmente ao usuário do serviço, quanto nas ações de saúde coletiva para comunidade. Essa nova realidade tecnológica, no procedimento de coleta de informações sobre o indivíduo, resulta em dados de maior confiabilidade para gestão, geração de conhecimento e, consequentemente, controle social. A atualização nos serviços de saúde é um processo ininterrupto e o acesso aos dados e informações de cada usuário do serviço, de maneira rápida e fácil, tem sido cada vez mais indispensável para os profissionais dessa área, aprimorando assim a assistência prestada ao indivíduo ${ }^{1-3}$.

No âmbito da coleta de informações e dados dos usuários estão inseridas as fichas de avaliação em fisioterapia. Observa-se a ausência de um software com um olhar mais holístico, tendo uma visão mais global baseada na Política Nacional de Saúde Funcional (PNSF). Aprovada em novembro de 2007, na 13 a Conferência Nacional de Saúde, a PNSF é ancorada no contexto de funcionalidade e incapacidade que os sujeitos podem sofrer na presença, ou mesmo, na ausência, de doenças, no momento em que são atendidos, visando potencializar as atividades funcionais, diminuir limitações e evitar a restrição de sua participação social3.

Os softwares em saúde visam melhorar a assistência prestada pelo serviço, com base em uma maior disponibilidade de informações sobre os pacientes e um acesso mais amplo aos dados armazenados, mais atualizados e legíveis. Com essa tecnologia, tem-se também: aumento da segurança dos usuários do serviço; possibilidade de comparar computacionalmente resultados de exames e a evolução do tratamento, auxiliando o diagnóstico e o plano assistencial; capacidade de rápido compartilhamento de informações, quando necessário; geração de relatórios e indicadores de gestão e assistenciais, tanto para fins epidemiológicos quanto estatísticos, colaborando para os processos de acreditação, entre outros ${ }^{4}$.

Com o objetivo de ampliar a investigação sobre os aspectos de saúde, a Organização Mundial da Saúde (OMS) lançou, em 2001, após mais de 25 anos de desenvolvimento, a Classificação Internacional de Funcionalidade, Incapacidade e Saúde (CIF). Os dados gerados pelo uso desta classificação poderão descrever o estado de funcionalidade como componente de saúde da população, permitindo conhecer amplamente o estado de saúde, a partir dos dados de morbimortalidade, admitindo uma abordagem terapêutica mais abrangente, mas também, atendendo a um número maior de necessidades do indivíduo 5 .

A CIF é um novo sistema de classificação, inserido na família de Classificações Internacionais da Organização Mundial de Saúde, constituindo o quadro de referência universal adotado pela OMS para descrever, avaliar e medir a saúde e a incapacidade tanto no nível individual como no populacional. Essa classificação possui múltiplas finalidades e pode ser utilizada de forma transversal em diferentes áreas disciplinares e setores como: saúde, educação, segurança social, emprego, economia, política social, desenvolvimento de políticas e da legislação em geral e alterações ambientais ${ }^{6}$.

A utilização da CIF pelos profissionais envolvidos no processo de reabilitação aumenta a qualidade e a individualidade dos dados relativos aos usuários. Duas pessoas com a mesma doença podem apresentar diferentes manifestações funcionais e duas pessoas com capacidade funcional equivalente não necessariamente apresentam a mesma condição de saúde. Ela é capaz de auxiliar a avaliação fisioterapêutica e o diagnóstico cinético-funcional do indivíduo, propiciando ao profissional uma visão mais global do usuário do serviço de saúde em todas as linhas de cuidado.

No intuito de promover o uso da CIF pelos profissionais de saúde e contribuir para o processo de informatização e coleta de dados dos usuários, a pesquisa aqui apresentada construiu um software avaliativo, 
que visa atender à linha de cuidado Saúde do adulto em fisioterapia ortopédica e traumatológica, na ótica da PNSF, e é capaz de registrar os dados do usuário do serviço através de um prontuário eletrônico funcional, inserindo as informações em um banco de dados virtual, formando assim um sistema de informação em saúde na fisioterapia.

\section{Caracterizando o problema}

Um processo comum que ocorre nos sistemas de informação em saúde é o uso do papel como fonte coletora primária de informações e de outros exames anexados. Além da efemeridade do material, a informação desse tipo de prontuário está disponível somente a um profissional por vez, e está sujeito à ilegibilidade, ambiguidade, perda de informação, multiplicidade de fichas do mesmo paciente, não padronização, dificuldade de acesso e, ainda, a um grande espaço para arquivo próprio ${ }^{8}$.

Esse método de coleta duplica o trabalho de registro rotineiro de dados. A digitação posterior desses dados aumenta a probabilidade de erros e vieses nas informações produzidas e utilizadas para a tomada de decisão .

Observando, através de uma ótica sustentável, esse uso indiscriminado de papel na coleta de informações sobre o usuário acarreta um problema para os gestores em saúde, que deverão preocupar-se com o desenvolvimento sustentável do país, ou seja, com um desenvolvimento que atenda às necessidades do presente (registro de informações), sem comprometer a possibilidade das futuras gerações de atender às suas próprias ${ }^{10}$.

Além disso, a aplicação da CIF pelos profissionais da saúde é algo complexo diante da sua dimensão, e observa-se, cada vez mais, a necessidade de estudos que mostrem a aplicação prática com os usuários, revelando-se uma nova ferramenta no cuidado da saúde do indivíduo ${ }^{11}$.

\section{Metodologia}

A informática associada à saúde tem-se mostrado bastante eficaz, contribuindo para otimizar o tempo e facilitar tarefas, principalmente no que diz respeito ao sistema de informação nessa área ${ }^{12}$.

A pesquisa aqui apresentada tem caráter exploratório e inclui a construção de um software de avaliação baseado na Classificação Internacional de Funcionalidade (CIF), nas áreas de fisioterapia ortopédica e traumatológica. Visa propor novos métodos e técnicas de coleta e armazenamento de informações dos usuários em clínicas-escolas de fisioterapia para inserção do prontuário eletrônico funcional como forma de registro do usuário do serviço de saúde.

O desenvolvimento desse projeto foi realizado na Fábrica de software do Centro Universitário de João Pessoa - Unipê, tendo sido iniciado em setembro de 2012 e finalizado em junho de 2016. Trata-se de um projeto integrado com a participação de docentes dos cursos de fisioterapia, ciência da computação e discentes dos cursos de fisioterapia, sistemas para internet, gestão em tecnologia da informação e ciência da computação.

\section{Instrumentos e construção do software}

Oprontuário a ser informatizado foi construído baseado na Classificação Internacional de Funcionalidade. A equipe o denominou CIFisio, e ele foi montado por meio de Core Sets, que são listas resumidas de categorias relevantes da CIF para doenças e contextos específicos ${ }^{13}$. O Ministério da Saúde estabeleceu o projeto de linhas de cuidado nas redes de atenção à saúde fundamentado no Artigo 198 da Constituição de 1988 com o propósito de romper com a fragmentação da atenção às necessidades sociais de cuidado dos usuários ${ }^{14}$. O software construído visa reduzir ações e serviços de saúde centrados em procedimentos, atos desconexos, compartimentados e fragmentados. 
O sistema foi desenvolvido em linguagem PHP, uma tecnologia open source que permite criar conteúdos dinâmicos para a web ${ }^{14}$ com framework CakePHP, uma abstração que une códigos comuns, o que torna a construção de aplicações web mais simples, mais rápidas, requerendo menos códigos e oferecendo um rápido acesso ao banco de dados ${ }^{15}$. Foi utilizado o banco de dados MySQL, com interfaces responsivas.

O CIFisio foi desenvolvido usando o modelo evolucionário da engenharia de software, o qual propõe a participação ativa do usuário durante o processo de desenvolvimento, assegurando um produto adequado às necessidades propostas. Este modelo assegura um processo com níveis elevados de refinamento do sistema através de múltiplos ciclos de análise, projeto, implementação, teste e homologação. O desenvolvimento do CIFisio buscou contemplar as recomendações do programa para Melhoria de Processo do Software Brasileiro (MPS.BR). O MPS.BR é coordenado pela Associação para Promoção da Excelência do Software Brasileiro (Softex). Segundo o Instituto Nacional de Metrologia, Qualidade e Tecnologia (Inmetro), essa associação é uma entidade privada, sem fins lucrativos, que objetiva incentivar estudos e pesquisas no seu observatório digital nacional, visando aumentar a competitividade entre as empresas produtoras de software e, através desta ação, melhorar a produção e qualidade da produção digital nacional ${ }^{16,17}$.

Com o levantamento de recursos e a fase de definição das funcionalidades do software, foi elaborada a estrutura analítica do projeto (EAP), que é uma ferramenta de decomposição do trabalho do software em partes manejáveis, ou seja, as suas funcionalidades.

Aplicou-se a modelagem de UML (Unified Modeling Language) ou Linguagem de Modelagem Unificada, que é uma linguagem gráfica de modelagem para visualizar, especificar, construir, documentar e comunicar artefatos e sistemas complexos. Ela permite descrever detalhadamente todos os casos de uso do software.

O CIFisio foi criado para ser utilizado por três tipos de usuários: (1) administrador; (2) docente; e (3) discente. Ele foi programado para ser manuseado em clínicas-escolas de fisioterapia, onde os docentes são administradores e controladores do fluxo de atendimentos e os discentes estagiários prestam assistência fisioterapêutica.

O administrador do sistema é o responsável por todas as informações coletadas pelos usuários. Ele é responsável pela administração dos dados, geração de relatórios e modelagem de decisão em saúde através das informações colhidas. Ele tem acesso a todas as informações contidas no programa após terem sido registradas pelos docentes e discentes. O docente é o administrador das informações coletadas pelos discentes, ele conhece as interfaces do programa, seu funcionamento e treina os seus usuários, validando as informações coletadas por eles. O acadêmico é o usuário do sistema. Ele é capaz de cadastrar novos usuários dos serviços de saúde, registrar a sua avaliação fisioterapêutica, os objetivos do tratamento, a conduta fisioterapêutica, os recursos utilizados em cada atendimento e as reavaliações.

Essa divisão de funções no programa foi criada para evitar erros e possíveis dados incompletos nos prontuários, aumentando a segurança do sistema e tornando o banco de dados mais protegido e confiável.

Na Figura 1 é possível visualizar a modelagem desse processo: 


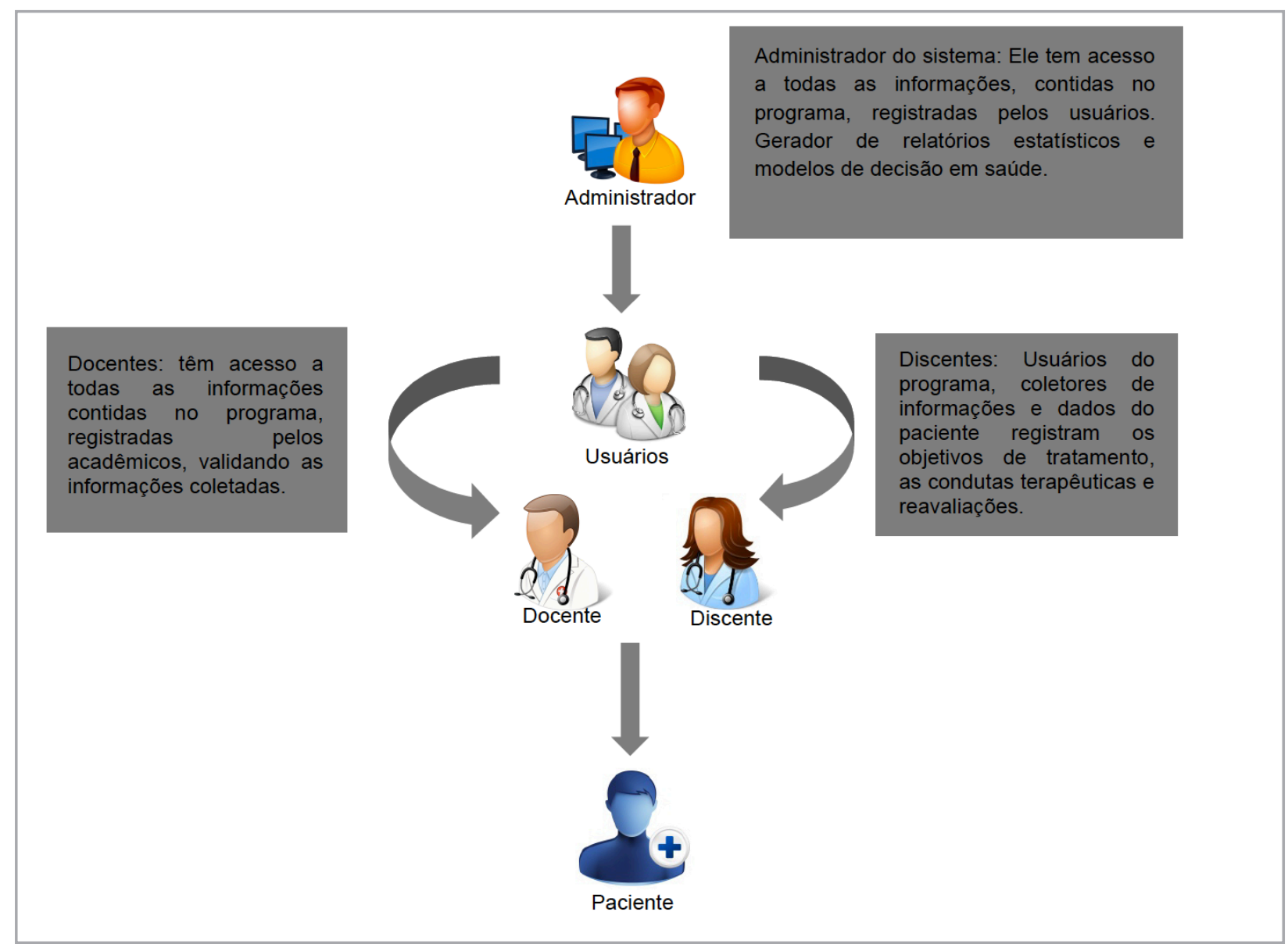

Figura 1. Diagrama de casos de uso

Fonte: Elaborada pelos autores, com base nos dados da pesquisa (2017).

A organização e a estrutura dos componentes significativos do sistema que interagem por meio das interfaces são chamadas de arquiteturas de software. Para o CIFisio, foi elaborado um diagrama arquitetural, um modelo arquitetural interno e externo para que os desenvolvedores pudessem visualizar, planejar e executar melhor a funcionalidade do sistema por meio dessas interfaces como mostram as Figuras 2, 3 e 4:

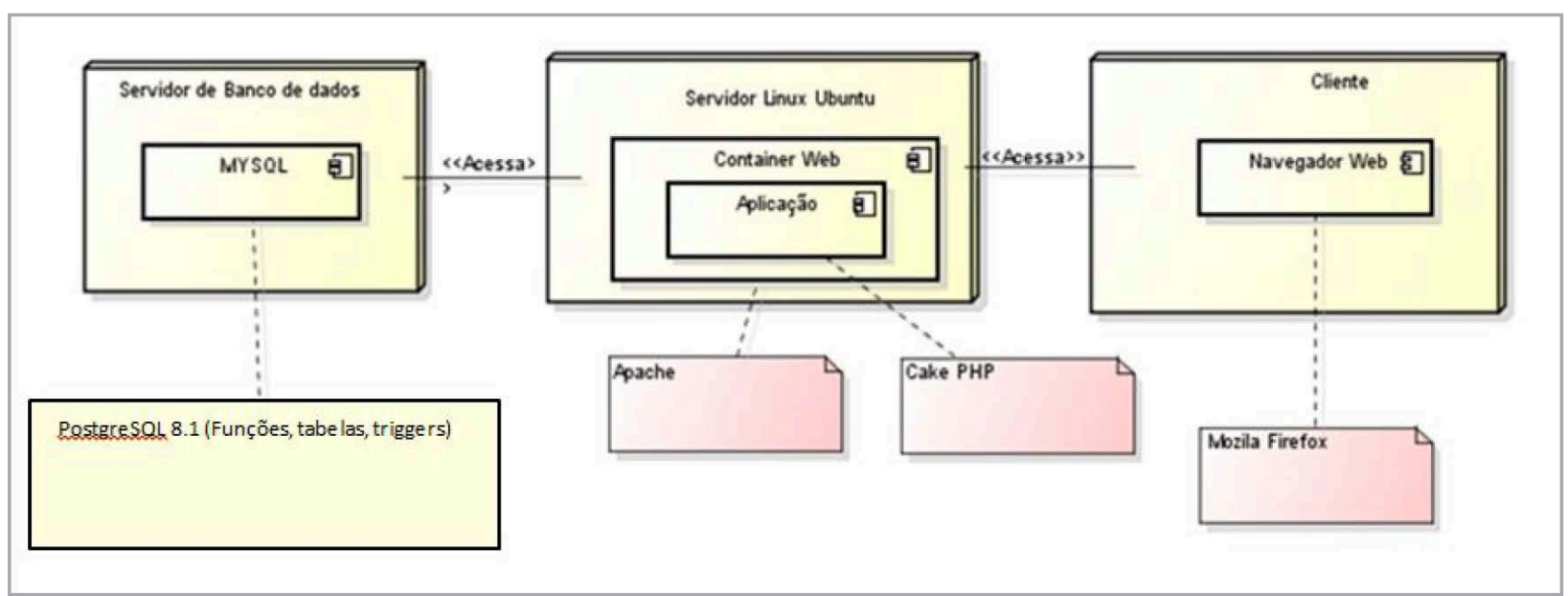

Figura 2. Diagrama arquitetural do CIFisio

Fonte: Arquitetura de software do Unipê (2017). 


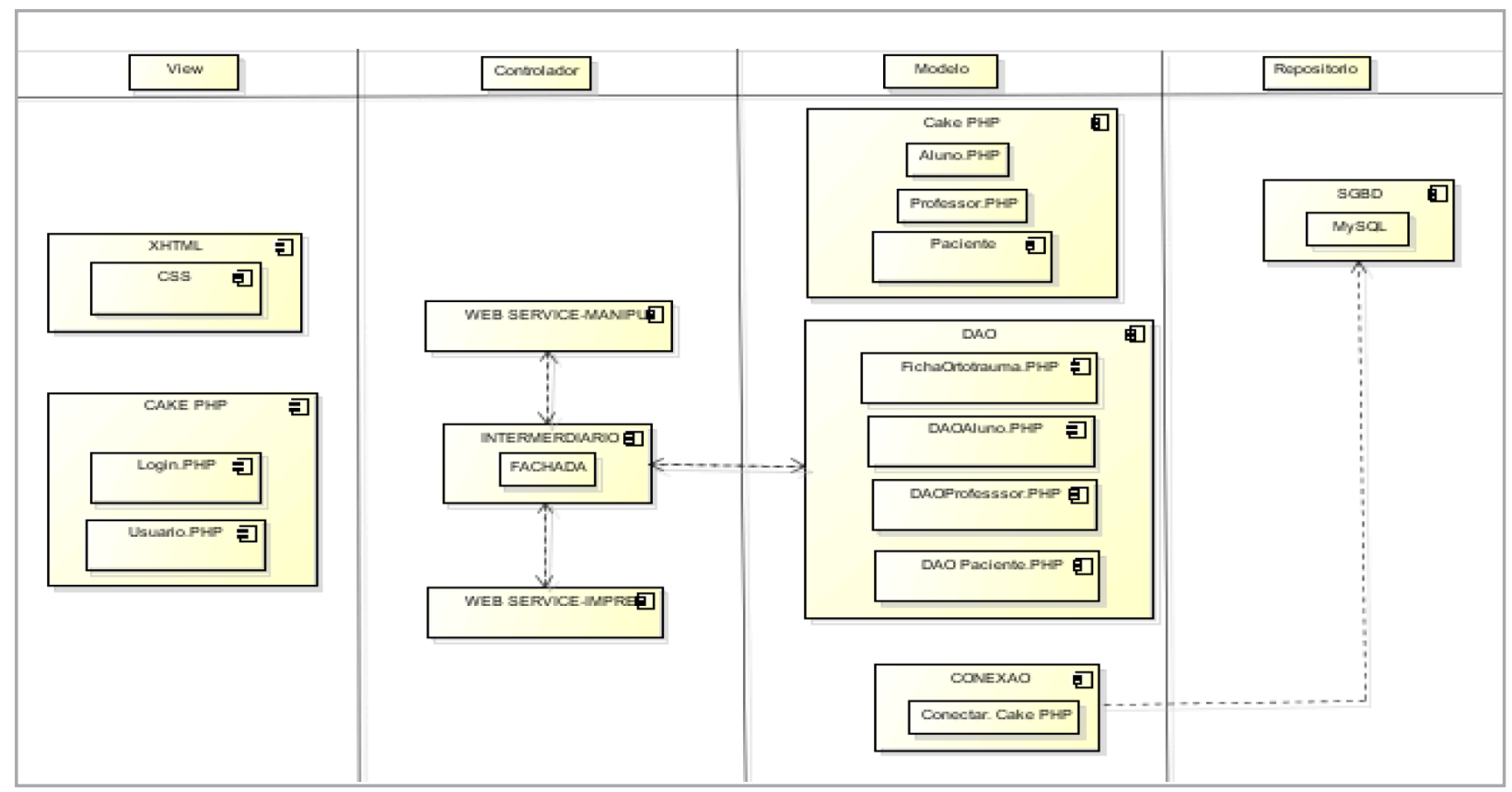

Figura 3. Modelo arquitetural interno

Fonte: Arquitetura de software do Unipê (2017).

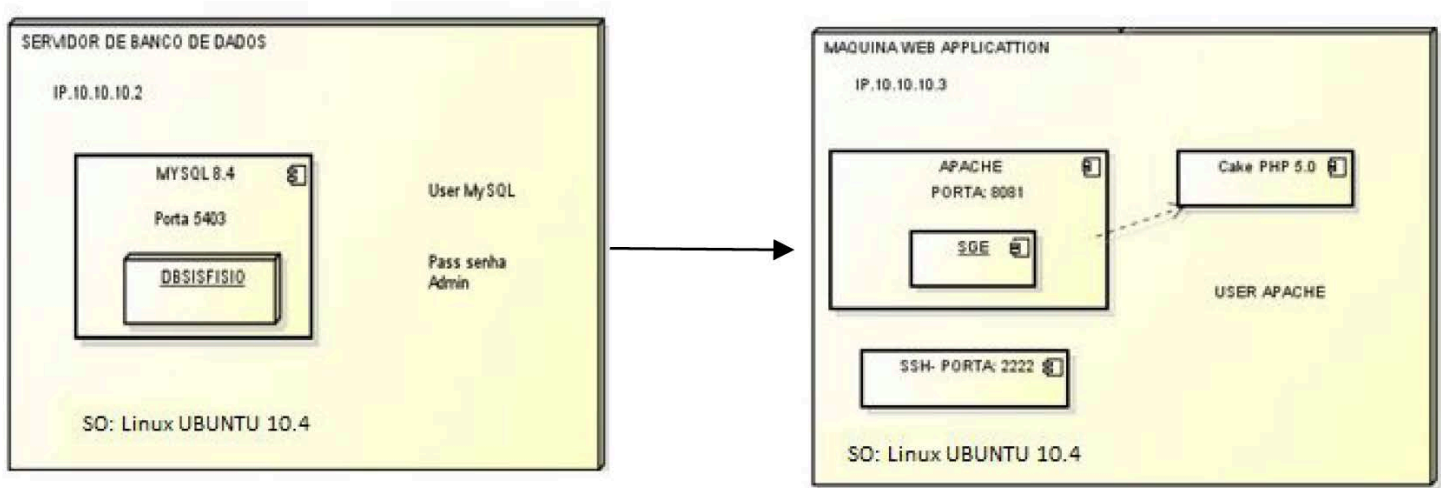

Figura 4. Modelo arquitetural externo

Fonte: Arquitetura de software do Unipê (2017).

O prontuário foi programado para registrar informações demográficas e epidemiológicas (nome, sexo, data de nascimento, endereço, formação escolar, estado civil, ocupação atual, diagnóstico médico das condições principais de saúde de acordo com a Classificação Internacional de Doenças CID-10, queixa principal e história da doença atual), como mostra a interface na Figura 5: 


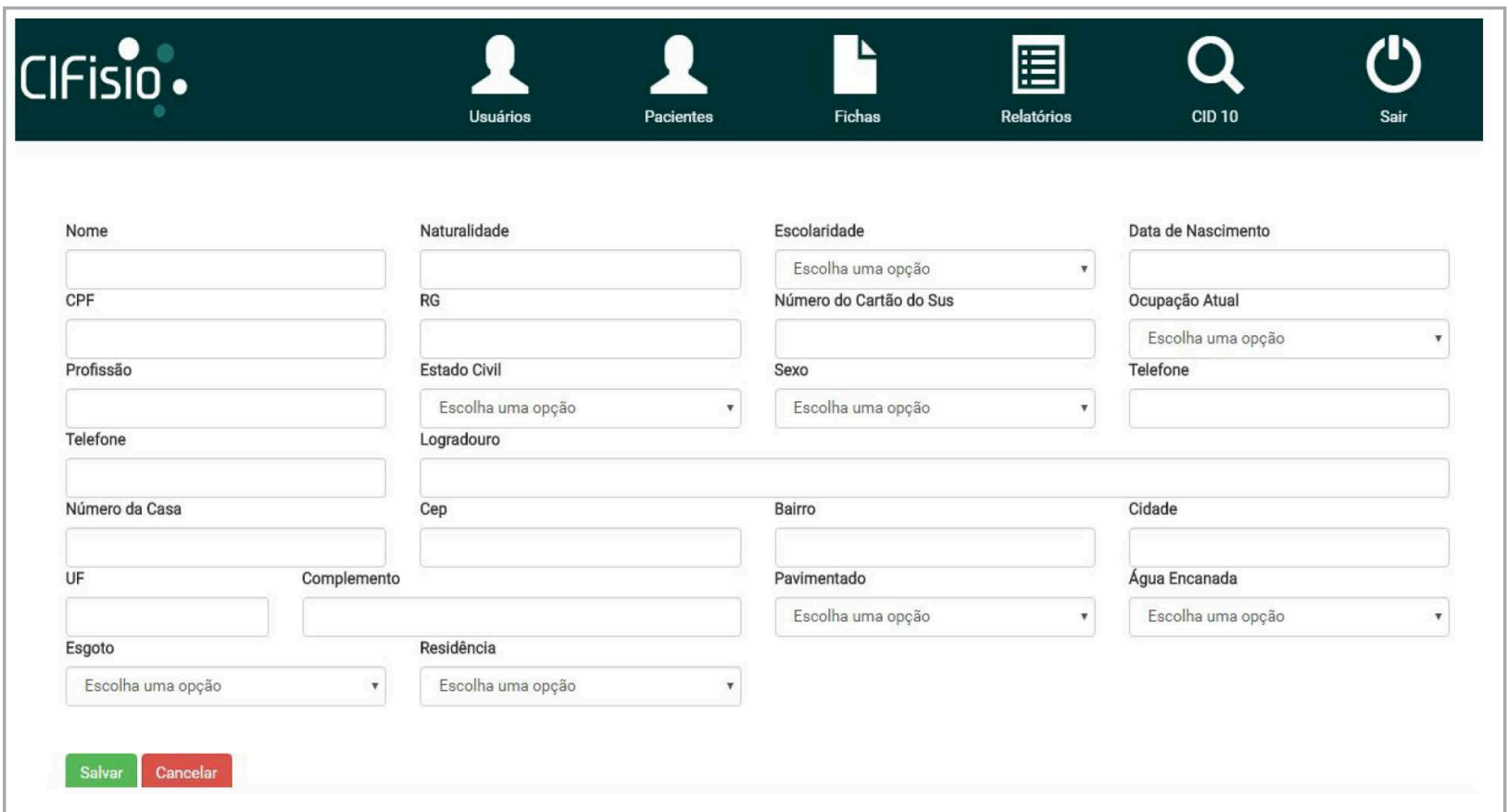

Figura 5. Interface inicial de cadastramento de pacientes

Fonte: Elaborada pelos autores com instrumental da Fábrica de softwares do Unipê (2017).

A função física e a cinética funcional serão avaliadas no software através da Classificação Internacional de Funcionalidades, fazendo uso das seguintes categorias: funções do corpo, estruturas do corpo, atividades e participação. Estas categorias estão organizadas por meio de alfabetos alfanuméricos dos quais fazem parte as letras B, D e E. A mensuração do grau de comprometimento será registrada através de qualificadores. Para as funções do corpo (B), por exemplo, foi utilizado o primeiro qualificador da CIF, que diz respeito à extensão das deficiências, sendo: o 'nenhuma deficiência': significa que a pessoa não tem problema; 1 'deficiência leve': significa um problema que está presente menos que $25 \%$ do tempo, com uma intensidade que a pessoa pode tolerar e que ocorrera nos últimos 30 dias; 2 'deficiência moderada': significa um problema que está presente menos da metade do tempo, com uma intensidade que interfere na vida diária da pessoa e que ocorrera ocasionalmente nos últimos 30 dias; 3 'deficiência grave': significa um problema que está presente mais da metade do tempo, com uma intensidade que prejudica/rompe parcialmente a vida diária da pessoa e que ocorrera frequentemente nos últimos 30 dias ${ }^{18}$.

O número 4 se refere à 'deficiência completa': significa um problema que está presente mais que $95 \%$ do tempo, com uma intensidade que prejudica/rompe totalmente a vida diária da pessoa e que ocorrera todos os dias nos últimos 30 dias; 8 'não especificado': significa que a informação é insuficiente para especificar a gravidade da deficiência; 9 'não aplicável': significa que é inapropriado aplicar um código particular. O Core Set para avaliar as funções do corpo foi composto por: funções sensoriais e dor; funções sensoriais relacionadas com a temperatura e outros estímulos; avaliação da dor; frequência cardíaca; sistema imunológico; mobilidade articular; estabilidade articular; mobilidade dos ossos; força; e resistência muscular ${ }^{19}$. A Figura 6 mostra um exemplo de como são avaliadas, no software, as funções relacionadas ao movimento. 


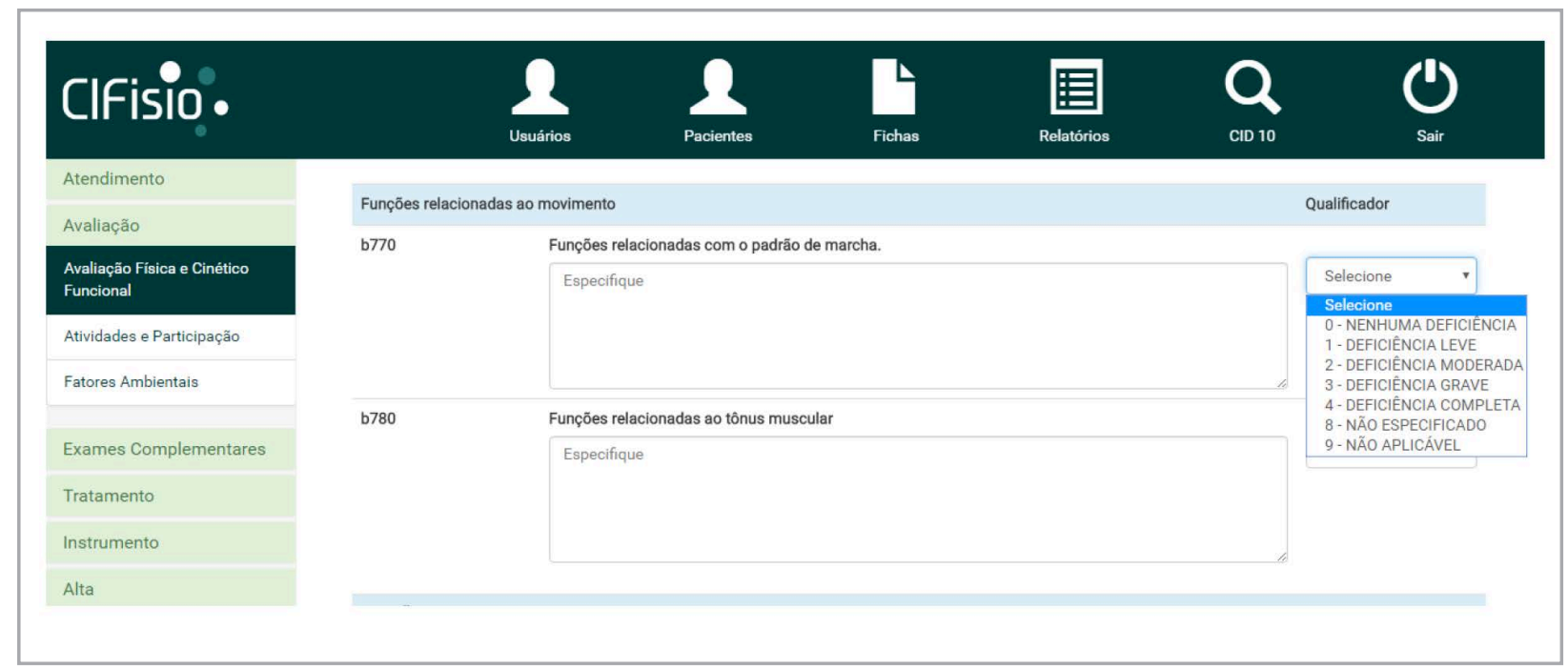

Figura 6. Interface de avaliação física e cinético-funcional do CIFisio

Fonte: Elaborada pelos autores com instrumental da Fábrica de softwares do Unipê (2017).

Quanto às atividades e participação (D), foi programado um campo no qual o usuário registra a avaliação das limitações e restrições encontradas no indivíduo. Neste campo, o avaliador poderá fazer uso de dois qualificadores para mensurar quanto as atividades de vida diária estão prejudicadas devido à sua afecção ortopédica ou traumatológica. O primeiro qualificador nessa categoria avalia a qualidade do desempenho das atividades e pode ser o mesmo qualificador utilizado pelas funções do corpo, descrito anteriormente. $\mathrm{O}$ segundo qualificador avalia a capacidade (sem ajuda) de o indivíduo realizar alguma atividade. Seus scores são: o - nenhuma dificuldade; 1 - dificuldade ligeira; 2 - dificuldade moderada; 3 - dificuldade grave; 4 - dificuldade completa; 8 - não especificada e 9 - não aplicável ${ }^{20}$. A Figura 7 exemplifica a tela descrita, mostrando o campo para o preenchimento desses dois qualificadores:

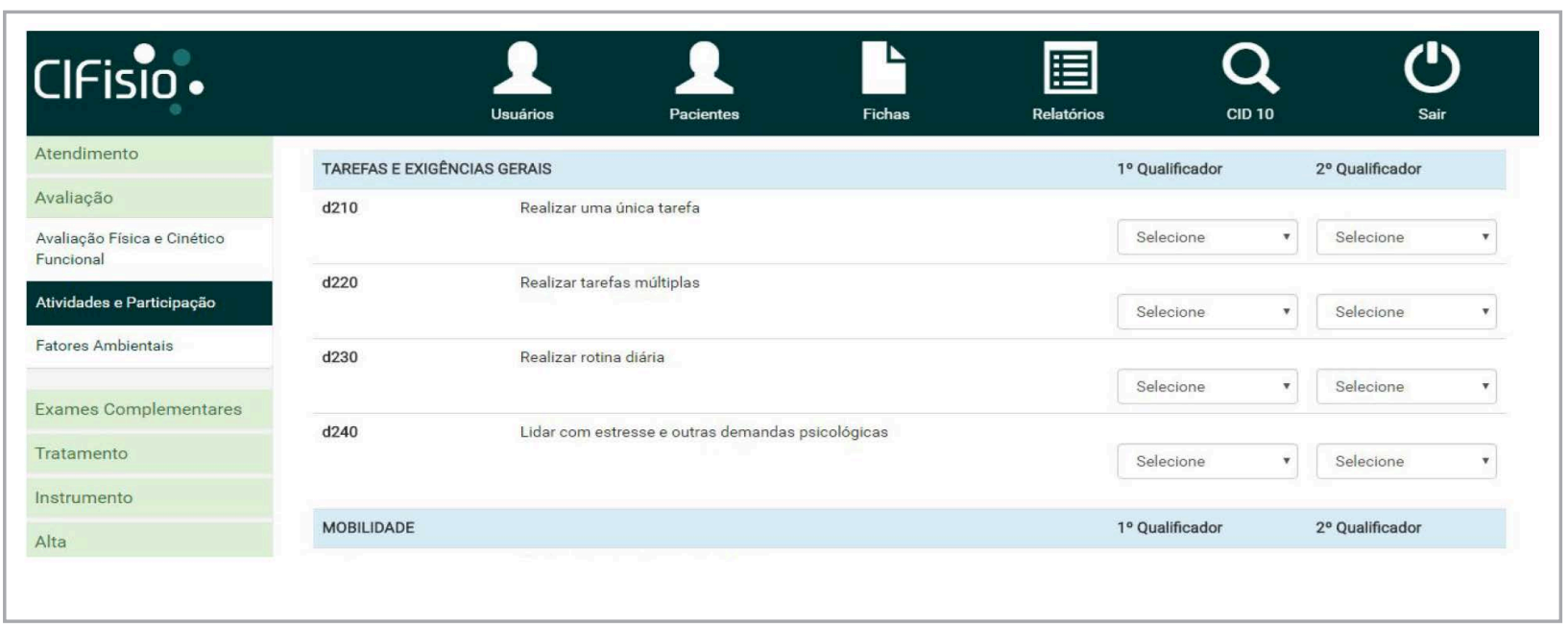

Figura 7. Interface de avaliação física e cinético-funcional do CIFisio

Fonte: Elaborada pelos autores com instrumental da

Fábrica de softwares do Unipê (2017).

Como mostra a Figura 8, na avaliação dos fatores ambientais (E), segundo a CIF, o score de mensuração é o qualificador de barreiras e facilitadores, sendo: o - nenhuma barreira; 1 - barreira leve; 2 - barreira moderada; 3 - barreira grave; 4 - barreira completa; o - nenhum facilitador; +1 - facilitador leve; +2 facilitador moderado; +3 - facilitador considerável; e +4 - facilitador completo ${ }^{18}$. 


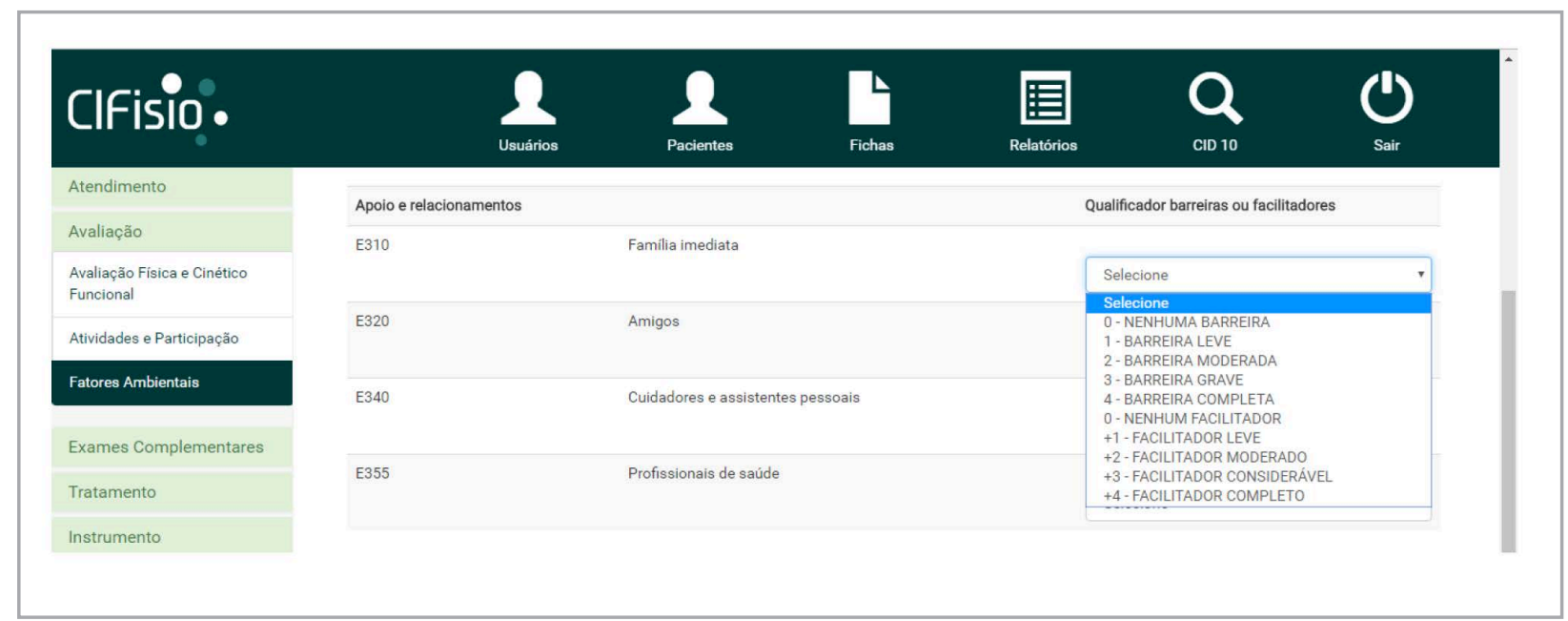

Figura 8. Interface de avaliação dos fatores ambientais do CIFisio

Fonte: Elaborada pelos autores com instrumental da Fábrica de softwares do Unipê (2017).

\section{Conclusão}

Esse software foi construído para informatizar a clínica-escola de fisioterapia do Unipê, servindo como prontuário eletrônico nos atendimentos de ortopedia e traumatologia para armazenamento dos dados do usuário do serviço, com o diferencial de ser uma coleta de dados multidimensional, trazendo uma visão global do usuário. O CIFisio armazena não só dados básicos de um prontuário eletrônico, como nome, endereço, testes específicos e diagnóstico, mas também fatores ambientais e psicossociais que influenciam o processo de saúde-doença, a recuperação do indivíduo e o retorno às suas atividades funcionais, além de ser um recurso didático para docentes e discentes no processo de ensino acadêmico.

Tal recurso pode ser um grande auxiliador na rotina da clínica-escola, facilitando o modo de registro dos prontuários, aprimorando a avaliação fisioterapêutica, devido à substituição do registro manual dos dados dos usuários pela possibilidade de armazenamento eletrônico, otimizando o tempo, aumentando a confiabilidade das informações coletadas na avaliação e na conduta diária, evitando assim: adulterações nas informações contidas nos prontuários, extravios e perdas dos dados com o passar dos anos ${ }^{21}$.

Além disso, é um software com iniciativa sustentável, pois as fichas de avaliações fisioterapêuticas baseadas na CIF são complexas e extensas, fazendo uso de um elevado número de folhas de papel. O CIFisio evitará o uso excessivo de papel, por possibilitar a realização das suas funções digitalmente, trazendo contribuições não só para a instituição, mas para toda a comunidade.

Portanto, o processo de digitalização é fundamental para a produção de relatórios, identificação de indicadores de saúde e melhor gestão da linha de cuidado saúde do adulto e da clínica-escola no processo do cuidar do usuário do serviço²2.

O desconhecimento de outra ferramenta que reúna todos os componentes do CIFisio o torna um software pioneiro e que faz jus ao aperfeiçoamento para que possa ser utilizado no ambiente operacional para o qual foi desenvolvido (atendimento ambulatorial). Dessa forma, vale ressaltar que a validação do CIFisio está em conformidade com processos de normas de desenvolvimento de software em sua utilização e avaliação fora do ambiente operacional. Entretanto, ainda deve passar por uma fase de utilização e aplicação dentro dessas unidades ambulatoriais. Cabe como recomendação para estudos futuros a avaliação de margens de erro, de confiabilidade e usabilidade do CIFisio mediante a utilização e avaliação do programa por profissionais fisioterapeutas diante de situações reais nas unidades ambulatoriais. 


\section{Referências}

1. Bakker AR. The need to know the history of the use of digital patient data, in particular the EHR. Int J Med Inform [Internet]. 2007 May-Jun[cited 2017 Aug 09];76(5-6):438-41. Disponível em: http://www. sciencedirect.com/science/article/pii/S1386505606002140

2. Ministério da Saúde, Secretaria Executiva, Departamento de Monitoramento e Avaliação do SUS (BR). Política Nacional de Informatização e Informática em Saúde. Brasília (DF); 2003.

3. Cavalcante RB, Silva PC, Ferreira MN. Sistemas de informação em saúde: possibilidades e desafios. Rev enferm UFSM[Internet]. 2011 [citado 2017 ago 09];1(2):290-9. Disponível em: https://periodicos.ufsm. br/reufsm/article/view/2580/1643

4. Lourenção LG; Ferreira Junior CJ. Implantação do prontuário eletrônico do paciente no Brasil. Enfermagem Brasil. 2016;15(1):44-53.

5. Ministério da Saúde (BR). Política Nacional de Saúde Funcional. Brasília(DF); 2011.

6. Araujo ES. A classificação internacional de funcionalidade, incapacidade e saúde (CIF) em fisioterapia: uma revisão bibliográfica[dissertação]. São Paulo:USP; 2008.

7. Fraga HM, Souza CS, Oliveira KGF. Os debates ancestrais e atuais acerca do que é saúde e classificação internacional de funcionalidade, incapacidade e saúde: contribuições para compreensão das possibilidades de uma Política Nacional de Saúde Funcional[Internet]. 2011 [citado 2016 out 13];1(1):1-11. Disponível em: https://goo.gl/JCpUwV

8. Maia RS,Santos LMA, Mendes NS, Barbosa AS, Oliveira RFR. Uso do prontuário eletrônico em Estratégia de Saúde da Família/Montes Claros-relato de experiência. R Intercâmbio[Internet]. 2016 [citado 2016 out 13];7:404-10. Disponível em: https://goo.gl/wyNEKi

9. Gutierrez MA. Sistemas de Informação Hospitalares: progressos e avanços. JHealth Inform [Internet]. 2011 [citado 2011ago 26];3(2):115-7.Disponível em: http://www.jhi-sbis.saude.ws/ojs-jhi/index.php/jhisbis/article/view/155/64

10. Lima DFB,BragaALS, FernandesJL, BrandãoES. Sistema de informação em saúde: concepções e perspectivas dos enfermeiros sobre o prontuário eletrônico do paciente. Rev Enf Ref [Internet]. 2011 dez [citado 2016out 04];5:113-9. Disponível em: https://goo.gl/1ZuvmA

11. Vall J, Costa CMC, Pereira LF, FriesenTT. Application of International Classification of Functioning, Disability and Health (ICF) in individuals with spinal cord injury. Arq Neuro-Psiquiatr [Internet]. 2011 Jun [citado em 20160ct03];69(3):513-8. Disponível em: https://goo.gl/jtVoaP

12. Marin HJ. Sistemas de Informação em Saúde: considerações gerais. J Health Inform [Internet]. 2010[citado em 2016 Oct. 03];2(1):20-4.Disponível em: https://goo.gl/a5XMt

13. Riberto M. Core sets da Classificação Internacional de Funcionalidade, Incapacidade e Saúde. RevBrasEnferm[Internet]. 2011 [citado 2016out 03];64(5):938-46. Disponível em: https://goo.gl/t5Pvb4

14. Tavares F. Desenvolvimento de aplicações em PHP. Lisboa: FCA; 2012.

15. Morais AM, Machado LS. Programação visual para um framework de desenvolvimento de aplicações médicas baseadas em realidade virtual. In: $12^{\circ}$ Congresso Brasileiro de Informática em Saúde; 2010out 18-22; Porto de Galinhas. Porto de Galinhas: [editora desconhecida]; 2010.

16. Conselho Regional de Fisioterapia e Terapia Ocupacional 5. Gestores Cuidando da Saúde do Povo [Internet]. 1 Folder. Porto Alegre, 2011 [citado em 2016 ago 25]. Disponível em: http://www.crefito5. org.br/wp-content/uploads/2011/07/PNSF.pdf

17. Pereira SR, Paiva PB. A importância da engenharia da usabilidade para a segurança de sistemas informatizados em saúde. J Health Inform [Internet]. 2011 [citado 2016out. 03];3(3):123-9. Disponível em: http://www.jhi-sbis.saude.ws/ojs-jhi/index.php/jhi-sbis/article/view/145/91.

18. Perez G, Zwicker R. Fatores determinantes da adoção de sistemas de informação na área de saúde: um estudo sobre o prontuário médico eletrônico. Rev Adm Mackenzie [Internet]. 2010[citado 2016 out. 04];11(1):174-200. Disponível em: https://goo.gl/PCTgUS.

19. Castaneda L, Bergmann A, Bahia L. A Classificação internacional de funcionalidade, incapacidade e saúde: uma revisão sistemática de estudos observacionais. Revbras epidemiol. [Internet]. 2014jun [citado 2016 out 03];17(2): 437-51. Disponível em: https://goo.gl/P3Rssa 
20. Fernández-López JA, Fernández-Fidalgo M, Geoffrey R, Stucki G, CiezaA. Funcionamiento y discapacidad: la clasificación internacional del funcionamiento (CIF). Rev Esp Salud Publica [Internet]. 2009 [citado 2016out 04];83(6):775-83. Disponível em: https://goo.gl/6mgoMN

21. Carreno I, Moreschi C, Marina B, Hendges DJB, Rempel C, Oliveira MMC. Análise da utilização das informações do Sistema de Informação de Atenção Básica (SIAB): uma revisão integrativa. Ciênc. Saúde Coletiva [Internet]. 2015 mar [citado 2016 out03];20(3):947-56. Disponível em: https://goo.gl/zZ5Jbh

22. Pereira BS,Tomasi E. Instrumento de apoio à gestão regional de saúde para monitoramento de indicadores de saúde. Epidemiol Serv Saúde [Internet]. 2016jun [citado 2016out 03]; 25(2):411-8. Disponível em: https://goo.gl/yoRXN9 